\title{
INDEXES TO VOLUMES 56 AND 57 AUTHOR INDEX
}

Author

AAltonen, M. \& INKeRI, K.

Akrivis, Georgios D. \& Dougalis, Vassilios A.

Alexander, Roger

Alfeld, Peter \&

Sirvent, MARITZA

AMMAR, G. S.

ARNo, Steven

Atkinson, Kendall E. \& Sloan, IAN H.

AzIz, A. K. \& LiU, J.-L.

BLECKSMITH, RICHARD, BRILLHART, JOHN \& Gerst, Irving

BRAmble, James H., PAsciak, Joseph E., WANG, JUNPING \& Xu, Jinchao

Bramble, James H., PASCIAK, Joseph E., WANG, JUNPING \& Xu, Jinchao

Bramble, James H., Pasciak, Joseph E. \& Xu, Jinchao

Bramble, James H. \& XU, Jinchao

Brent, R. P., Cohen, G. L. \& TE Riele, H. J. J.

BRIgGS, KeITH

BRILlHART, JOHN

Calvetti, Daniela

Carlson, B. C.

Chern, I-Liang

Chou, So-Hsiang \& LI, QIAN
Title

Catalan's equation $x^{p}-y^{q}=1$ and related congruences ............... 56

Finite difference discretizations of some initial and boundary value problems with interface . . .

The modified Newton method in the solution of stiff ordinary differential equations .....

The structure of multivariate superspline spaces of high degree . . . . . . . . . . 57

See: Reichel, L., Ammar, G. S. \& Gragg, W. B. 57

A note on Perrin pseudoprimes ........

The numerical solution of first-kind logarithmickernel integral equations on smooth open arcs

A Galerkin method for the forward-backward heat equation ............. 56

On a certain $(\bmod 2)$ identity and a method of proof by expansion ...........

Convergence estimates for product iterative methods with applications to domain decomposition

Convergence estimates for multigrid algorithms without regularity assumptions . . . . . . .

The analysis of multigrid algorithms with nonnested spaces or noninherited quadratic forms

Improved techniques for lower bounds for odd perfect numbers ............ 57

A precise calculation of the Feigenbaum constants

See: BleCKSMITH, RichaRd, BRILlHART, JohN \&

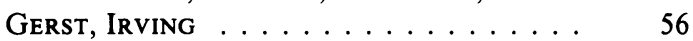

A stochastic roundoff error analysis for the Fast Fourier Transform ............ 56

A table of elliptic integrals: One quadratic factor

Large-time behavior of solutions of Lax-Friedrichs finite difference equations for hyperbolic systems of conservation laws . . . . . . . . . .

Mixed finite element methods for compressible miscible displacement in porous media . . . . 
Author

Collins, Charles \& LUSKIN, Mitchell

Colquitt, W. N. \&

WELSH, L., JR.

COOPER, G. J.

COQUel, Fredéric \&

LE FLOCH, PhILIPPE

Costello, Patrick

Cottet, Georges-Henri

Darmon, Henri

Dawson, Clint N.,

Du, QIANG \&

DUPONT, TODD F.

DIAZ Y DIAZ, F.

Disney, Shaun \&

SLOAN, IAN H.

Doll, Helmut \&

HOSTE, JIM

Dougalis, Vassilios A.

DOUGHeRTY, RANDALL \&

Janwa, HeEralal

DU, QIANG

Dummit, D. S.

DUPONT, TODD F.

EgGERMONT, P. P. B. \& LUBICH, $\mathrm{CH}$.

EICHENAUER-HERRMANN, JÜRGEN

Elman, Howard C. \& Golub, Gene H.

ERNVALL, R. \&

METSÄNKYLÄ, T.

EwING, R.

EwING, R. E.,

LAZAROV, R. D. \&

VASSILEVSKI, P. S.

FALK, RICHARD $S$. ture methods for nonlinear convolution equa-
Title

On the continuity in $\mathrm{BV}(\Omega)$ of the $L^{2}$-projection into finite element spaces . . . . . . . . .

See: Brent, R. P., Cohen, G. L. \& te Riele, H. J. J. . . . . . . . . . . . . . .

Vol. Page

Optimal-order error estimates for the finite element approximation of the solution of a nonconvex variational problem . . . . . . . . .

A new Mersenne prime ........... 56

$56 \quad 867$

On the implementation of singly implicit RungeKutta methods . . . . . . . . . .

Convergence of finite difference schemes for conservation laws in several space dimensions: the corrected antidiffusive flux approach . . . . .

Amicable pairs of the form $(i, 1) \ldots \ldots \ldots$

Large-time behavior of deterministic particle approximations to the Navier-Stokes equations

Note on a polynomial of Emma Lehmer . . . . .

A finite difference domain decomposition algorithm for numerical solution of the heat equa-

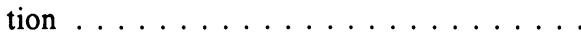

A table of totally real quintic number fields ...

Error bounds for the method of good lattice points

A tabulation of oriented links $\ldots \ldots \ldots \ldots 5$

See: Akrivis, Georgios D. \& Dougalis, VAssilios A. . . . . . . . . . .

Covering radius computations for binary cyclic codes ................

See: Dawson, Clint N., Du, Qiang \& Dupont,

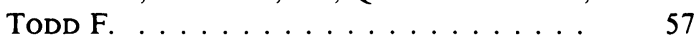

Solving solvable quintics . . . . . . . . . $\quad 57$

See: Dawson, Clint N., Du, Qiang \& Dupont, Todd F. . . . . . . . . . . . . .

Uniform error estimates of operational quadrations on the half-line . . . . . . . . . 56

Inversive congruential pseudorandom numbers avoid the planes . . . . . . . . . . . 56

Iterative methods for cyclically reduced non-selfadjoint linear systems. II . . . . . . . . . 56

Cyclotomic invariants for primes between 125000 and $150000 \ldots \ldots \ldots \ldots \ldots \ldots$

See: Lin, T., Sochacki, J., Ewing, R. \& GeORGE, J. . . . . . . . . . . . . . . . 56

Local refinement techniques for elliptic problems on cell-centered grids I. Error analysis . . . .

Nonconforming finite element methods for the equations of linear elasticity . . . . . . . 
FEe, GReg \&

GranVille, ANDREW

Fiorot, J. C. \& TABKA, J.

Fischer, BERND \&

Golub, Gene H.

FlammenKamp, ACHIM

DE Frutos, J.,

ORTEGA, T. \&

SANZ-SERnA, J. M.

Funaro, Daniele \&

Gottlieb, David

Funaro, Daniele \&

Kavian, OtaRed

GARCIA-ARCHILla, B. \&

SANZ-Serna, J. M.

GAUtsChi, WALTER

GAUtschi, WALTER

George, J.

GERST, IRVING

Glassey, Robert \&

SCHAEFFER, JACK

Goldstein, Charles I.

Gollan, Holger W.

Golub, Gene H.

Golub, Gene H.

Gordon, Daniel M. \& POMERANCE, CARL

Gottlieb, David

Gottlieb, David \& TADMOR, EITAN

GRAGG, W. B.

GranVille, ANDREW

GunzBuRger, M. D., Hou, L. \&

SvobodNY, T. P.

Gunzburger, Max D., MEIR, AMNON J. \&

Peterson, Janet $S$.
The prime factors of Wendt's binomial circulant determinant ............ 57

Shape-preserving $C^{2}$ cubic polynomial interpolating splines

On generating polynomials which are orthogonal over several intervals . . . . . . . . .

New sociable numbers . . . . . . . . . . . . 56

Pseudospectral method for the "good" Boussinesq equation ................ 57

Convergence results for pseudospectral approximations of hyperbolic systems by a penaltytype boundary treatment .........

Approximation of some diffusion evolution equations in unbounded domains by Hermite functions . . . . . . . . . . . . . .

A finite difference formula for the discretization of $d^{3} / d x^{3}$ on nonuniform grids . . . . . .

A class of slowly convergent series and their summation by Gaussian quadrature . . . . . . . .

On certain slowly convergent series occurring in plate contact problems . . . . . . . . . .

See: Lin, T., Sochacki, J., EwING, R. \& George, J. . . . . . . . . . . . . .

See: BLECKSMITH, RichARD, BRILlHART, JOHN \& Gerst, IRVING . . . . . . . . . . . 56

Convergence of a second-order scheme for semilinear hyperbolic equations in $2+1$ dimensions

Multigrid analysis of finite element methods with numerical integration ........... 56

The 5-modular representations of the Tits simple group in the principal block ........ 57

See: Elman, Howard C. \& Golub, Gene H. 215

See: Fischer, Bernd \& Golub, Gene H. . . . .

The distribution of Lucas and elliptic pseudoprimes ............... 57

See: Funaro, Daniele \& Gottlieb, David . . 585

The CFL condition for spectral approximations to hyperbolic initial-boundary value problems

See: Reichel, L., Ammar, G. S. \& Gragg, W. B. 273

See: Fee, Greg \& Granville, Andrew . . . . . 839

Analysis and finite element approximation of optimal control problems for the stationary Navier-Stokes equations with distributed and Neumann controls . . . . . . . . . . .

On the existence, uniqueness, and finite element approximation of solutions of the equations of stationary, incompressible magnetohydrodynamics . . . . . . . . . . . . 
Author

Hagstrom, ThOMAS

Hasegawa, Takemitsu \&

TORII, TATSUO

Hayes, David R.

HosTE, JIM

Hou, L.

HuANG, Mingyou

Hung, David C.

INKERI, $\mathrm{K}$.

IVIĆ, A. \& TE Riele, H. J. J.

Jakubec, Stanislav,

Kostra, JURAJ \&

Nemoga, Karol

Janwa, Heeralal

KANEKo, Hideaki \&

Xu, Yuesheng

KaVian, OtARED

KeAst, P.

Kostra, Juraj

KRÖNER, DiETMAR

Kurita, Yoshiharu \&

MATSUMOTO, MaKoto

LASIECKA, I. \&

TRIGGIANI, R.

LAWRENCE, JIM

LAZAROV, R. D.

LECOT, ChristiaN

L'ECUYer, PIERRE \&

TezuKa, SHu

LE FLOCh, PhILIPPE

LENFERINK, H. W. J.

LENFERINK, H. W. J. \&

SPIJKER, M. N.

LENFERINK, H. W. J. \&

SPIJKER, M. N.

LENSTRA, H. W., JR.

LI, QIAN
Title

Vol. Page

Asymptotic boundary conditions for dissipative waves: General theory . . . . . . . . . .

An automatic quadrature for Cauchy principal value integrals . . . . . . . . . .

On the reduction of rank-one Drinfeld modules

See: Doll, Helmut \& Hoste, Jim . . . . . . .

See: GunZBURger, M. D., Hou, L. \& SvobodNy, T. P. . . . . . . . . . . . . . 57

A Hamiltonian approximation to simulate solitary waves of the Korteweg-de Vries equation . .

Even positive definite unimodular quadratic forms over $\mathbb{Q}(\sqrt{3}) \ldots \ldots \ldots \ldots \ldots \ldots \ldots$

See: Aaltonen, M. \& InKeri, K. . . . . . . . 56

On the zeros of the error term for the mean square of $\left|\zeta\left(\frac{1}{2}+i t\right)\right| \ldots \ldots \ldots \ldots \ldots$

On the existence of an integral normal basis generated by a unit in prime extensions of rational numbers ............... 56

See: Dougherty, Randall \& Janwa,

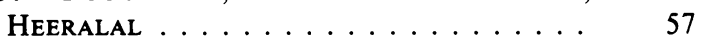

Degenerate kernel method for Hammerstein equations . . . . . . . . . . .

See: Funaro, Daniele \& Kavian, Otared . .

See: Lyness, J. N., Sørevik, T. \& Keast, P. . .

See: Jakubec, Stanislav, Kostra, Juraj \& Nemoga, Karol . . . . . . . . . . . .

Absorbing boundary conditions for the linearized Euler equations in 2-D . . . . . . . . .

Primitive $t$-nomials $(t=3,5)$ over $G F(2)$ whose degree is a Mersenne exponent $\leq 44497 \ldots$

Numerical approximations of algebraic Riccati equations for abstract systems modelled by analytic semigroups, and applications . . . . .

Polytope volume computation . . . . . . . . .

See: Ewing, R. E., Lazarov, R. D. \& Vassilev-

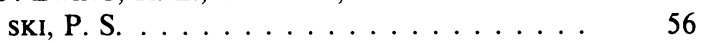

A quasi-Monte Carlo method for the Boltzmann

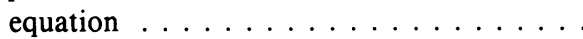

Structural properties for two classes of combined random number generators . . . . . . . .

See: Coquel, Frédéric \& Le Floch, Philippe

Contractivity-preserving implicit linear multistep

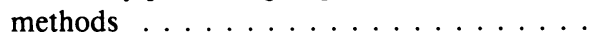

On a generalization of the resolvent condition in the Kreiss matrix theorem ......... 57

On the use of stability regions in the numerical analysis of initial value problems ......

Finding isomorphisms between finite fields ... 56

See: Chou, So-Hsiang \& Li, Qian . . . . . . 57 
Author

LI, T. Y. \&

WANG, XIAOSHEN

LiN, T., Sochacki, J.,

EWING, R. \& GEORGE, J.

LIU, J.-L.

LUBich, $\mathrm{CH}$.

LUSKIN, MitchelL

LyNess, J. N., SøreviK, T. \& Keast, $\mathrm{P}$.

Maiorana, James A.

Matsumoto, Makoto

Meir, AMnon J.

METSÄNKYLÄ, T.

Moews, David \&

Moews, Paul C.

Moews, Paul C.

Nemoga, Karol

Nochetto, R. H.,

PAOLINI, M. \&

VERDI, C.

Opfer, Gerhard

O'RIORDAN, Eugene

O'RIORDAN, EUGENE \&

STYNES, MARTIN

Ortega, T.

OWREN, BRYNJULF \&

ZenNaro, Marino

PAOLINI, M.

PASCIAK, Joseph E.

Pasciak, Joseph E.

PASCIAK, Joseph E.

Peterson, Janet S.

Pethö, Attila

Pomerance, CARL

RABINOWITZ, PhILIP
Title

Vol. Page

Solving deficient polynomial systems with homotopies which keep the subschemes at infinity invariant ............. 56

Some grid refinement schemes for hyperbolic equations with piecewise constant coefficients . . .

See: AzIz, A. K. \& LIU, J.-L. . . . . . . . . . . $56 \quad 56 \quad 35$

See: Eggermont, P. P. B. \& Lubich, Сн. . . . . $56 \quad 56149$

See: Collins, Charles \& Luskin, Mitchell . $57 \quad 621$

Notes on integration and integer sublattices . . . 56

A classification of the cosets of the Reed-Muller code $\mathscr{R}(1,6) \ldots \ldots \ldots \ldots \ldots$

See: Kurita, Yoshiharu \& Matsumoto, MAKOTO . . . . . . . . . . .

See: Gunzburger, Max D., Meir, Amnon J. \& Peterson, Janet S. . . . . . . . . . 56

See: Ernvall, R. \& Metsänkylä, T. . . . . . 56

A search for aliquot cycles below $10^{10} \ldots \ldots \quad 57$

851

849

See: Moews, David \& Moews, Paul C. . . . . 57

849

See: Jakubec, Stanislav, Kostra, Juraj \& Nemoga, Karol

An adaptive finite element method for two-phase Stefan problems in two space dimensions. Part I: Stability and error estimates . . . . . . . .

See: Reichel, Lothar \& Opfer, Gerhard . .

See: Stynes, Martin \& O'Riordan, Eugene .

A globally uniformly convergent finite element method for a singularly perturbed elliptic problem in two dimensions. . . . . . . . . . . .

See: de Frutos, J., Ortega, T. \& Sanz-Serna, J. $\mathrm{M}$.

Order barriers for continuous explicit Runge-Kutta methods ............. 56

See: Nochetto, R. H., Paolini, M. \& Verdi, C.

See: Bramble, James H., Pasciak, Joseph E., WANG, JunPing \& Xu, JinchaO . . . . . . .

See: Bramble, James H., Pasciak, Joseph E., Wang, JunPing \& Xu, Jinchao . . . . . . .

See: Bramble, James H., Pasciak, Joseph E. \& $\mathrm{Xu}, \mathrm{J}$ INCHAO

See: Gunzburger, Max D., Meir, Amnon J. \& Peterson, Janet S.

Complete solutions to families of quartic Thue equations . . . . . . . . . . .

See: Gordon, Daniel M. \& Pomerance, Carl

Uniform convergence results for Cauchy principal value integrals . . . . . . . . . . 56 
Author

ReICHEL, L., AMMAR, G. S. \& GRAGG, W. B.

REICHEL, LOTHAR \& OPFER, GERHARD

TE RIELE, H. J. J.

TE RiELE, H. J. J.

SaAvedra, Patricia \& SCOTT, L. RIDGWAY

SANZ-SERna, J. M.

Sanz-Serna, J. M.

SCHAEFFER, JACK

Schneider, Claus

ScotT, L. RIDGWAY

SiRvent, Maritza

SLOAN, IAN H.

SLOAN, IAN H.

SMYTH, C. J.

SOCHACKI, J.

SøREVIK, T.

SPIJKER, M. N.

SPIJKER, M. N.

STEIDL, G. \&

TASCHE, M.

Stynes, Martin

Stynes, Martin \& O'RIORDAN, EUGENE

SvobodNy, T. P.

TABKA, J.

TADMOR, EITAN

TASCHE, M.

TEMAM, $R$.

Tezuka, SHu

TORII, TATSUO

TRIGgIaNI, $R$.

TZANAKIS, N. \& DE Weger, B. M. M.

VALLEE, BrigitTe
Title

Vol. Page

Discrete least squares approximation by trigonometric polynomials . . . . . . . . . . 57 273

Chebyshev-Vandermonde systems . . . . . . . 57 703

See: Brent, R. P., Cohen, G. L. \& te Riele, H. J. J. . . . . . . . . . . . . . . . . . $57 \quad 857$

See: Ivić, A. \& TE Riele, H. J. J. . . . . . . . . $\quad 56 \quad 303$

Variational formulation of a model free-boundary problem .............. 57 451

See: de Frutos, J., Ortega, T. \& Sanz-Serna,

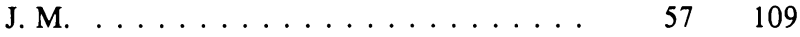

See: Garcia-Archilla, B. \& Sanz-Serna, J. M. $\quad 57 \quad 239$

See: Glassey, Robert \& SCHAEFFER, JACK . . $\quad 56 \quad 57$

Rosenbrock-type methods adapted to differentialalgebraic systems . . . . . . . . . . 56 56201

See: SaAvedra, Patricia \& Scott, L. Ridgway $57 \quad 451$

See: Alfeld, Peter \& Sirvent, Maritza $\ldots . .57 \quad 299$

See: Atrinson, Kendall E. \& Sloan, Ian H. . $\quad 56 \quad 119$

See: Disney, Shaun \& Sloan, Ian H. . . . . . $56 \quad 56257$

Ideal 9th-order multigrades and Letac's elliptic

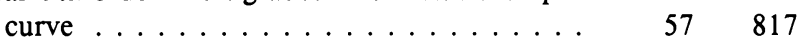

See: Lin, T., Sochacki, J., EwING, R. \& GEORGE, J. . . . . . . . . . . . . . . . $56 \quad 61$

See: Lyness, J. N., Sørevik, T. \& Keast, P. . . $\quad 56243$

See: Lenferink, H. W. J. \& SPIJKer, M. N. . . $\quad 57 \quad 211$

See: Lenferink, H. W. J. \& SPIJKer, M. N. . . $\quad 57221$

A polynomial approach to fast algorithms for discrete Fourier-cosine and Fourier-sine

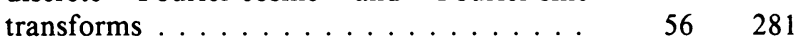

See: O'Riordan, Eugene \& Stynes, Martin . $\quad 57 \quad 47$

An analysis of a singularly perturbed two-point boundary value problem using only finite element techniques.............

See: Gunzburger, M. D., Hou, L. \& Svobodny,

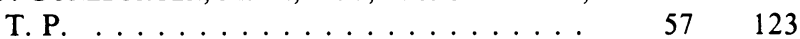

See: Fiorot, J. C. \& TABKA, J. . . . . . . . . $57 \quad 291$

See: GotTlieb, David \& TADMOR, Eitan . . . $\quad 56 \quad 565$

See: Steidl, G. \& TASche, M. . . . . . . . . . . $56 \quad 281$

Stability analysis of the nonlinear Galerkin method .............. $57 \quad 477$

See: L'Ecuyer, Pierre \& Tezuka, Shu . . . . $57 \quad 735$

See: Hasegawa, TAKemitsu \& ToriI, Tatsuo . 56741

See: Lasiecka, I. \& Triggiani, R. . . . . . . $\quad 57 \quad 639$

Solving a specific Thue-Mahler equation . . . . $57 \quad 57 \quad 799$

Generation of elements with small modular squares and provably fast integer factoring algorithms 
Author

VASERSTEIN, L. N.

VASSILEVSKI, P. S.

VERDI, C.

Viallon, Marie-Claude

Walker, Peter

WANG, JUNPING

WANG, JUNPING

WANG, JUNPING

WANG, XIAOSHEN

WASHINGTON, LAWRENCE C.

DE Weger, B. M. M.

WELSH, L., JR.

$\mathrm{XU}$, Jinchao

$\mathrm{Xu}$, JinchaO

$\mathrm{XU}$, JiNCHAO

$\mathrm{XU}$, JiNCHAO

$\mathrm{Xu}$, Jinchao

XU, YUESHENG

YE, QIANG

ZenNaro, Marino

\section{Title}

Vol. Page

Sums of cubes in polynomial rings $\ldots . \ldots$.

See: Ewing, R. E., LAzarov, R. D. \& VassilevSKI, P. S. . . . . . . . . . . . .

See: Nochetto, R. H., Paolini, M. \& Verdi, C.

Convergence of the two-point upstream weighting scheme . . . . . . . . . . .

Infinitely differentiable generalized logarithmic and exponential functions . . . . . . . .

See: Bramble, James H., Pasciak, Joseph E., Wang, JunPing \& Xu, Jinchao . . . . . .

See: Bramble, James H., Pasciak, Joseph E., WANG, JUNPING \& XU, JinchaO . . . . . . .

Superconvergence and extrapolation for mixed finite element methods on rectangular domains

See: LI, T. Y. \& WANG, XIAOShen ........

A family of cyclic quartic fields arising from mod-

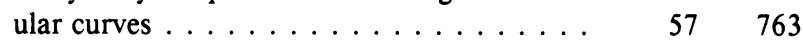

See: Tzanakis, N. \& DE Weger, B. M. M. . . . 57579

See: Colquitt, W. N. \& Welsh, L., JR. . . . . $\quad 56 \quad 567$

Counterexamples concerning a weighted $L^{2}$ projection ..............

See: Bramble, James H., Pasciak, Joseph E. \& Xu, Jinchao . . . . . . . . . . .

See: Bramble, James H., Pasciak, Joseph E., WANG, JunPING \& Xu, JinchaO . . . . . . .

See: Bramble, James H., Pasciak, Joseph E., WANG, JUNPING \& XU, JiNCHAO . . . . . . . $57 \quad 23$

See: Bramble, James H. \& Xu, Jinchao ... . $56 \quad 56463$

See: Kaneko, Hideaki \& Xu, Yuesheng . . . . $56 \quad 56141$

A convergence analysis for nonsymmetric Lanczos algorithms ............... $56 \quad 677$

See: OWren, Brynulf \& ZenNARo, Marino . $\quad 56 \quad 645$ 


\section{INDEX OF REVIEWS BY AUTHOR OF WORK REVIEWED}

\begin{tabular}{|c|c|c|c|c|}
\hline Author & $\begin{array}{l}\text { Review } \\
\text { Number }\end{array}$ & Classification & Vol. & Page \\
\hline $\begin{array}{l}\text { ABAFFY, JozSEF \& } \\
\text { SPEDICATO, EMILIO }\end{array}$ & 5 & $65-02,65 \mathrm{~F} 10,65 \mathrm{H} 10$ & 56 & 382 \\
\hline Allen, M. P. \& Tildesley, D. J. & 30 & $70-01,82-01,82 \mathrm{~A} 50,70-04,82-04$ & 57 & 442 \\
\hline An, Myoung & 25 & $\begin{array}{l}\text { See: Tolimieri, Richard, AN, } \\
\text { Myoung \& Lu, ChaO }\end{array}$ & 56 & 876 \\
\hline $\begin{array}{l}\text { BanK, R. E., Bulirsch, R. \& } \\
\text { MERTEN, K., Editors }\end{array}$ & 22 & $65-06,65 \mathrm{~L} 05,65 \mathrm{P} 05$ & 56 & 405 \\
\hline $\begin{array}{l}\text { BELINFANTE, JoHAN G. F. \& } \\
\text { Kolman, BERNARD }\end{array}$ & 28 & $17-01,17-04,22-01$ & 56 & 886 \\
\hline $\begin{array}{l}\text { Bergum, G. E., Philippou, A. N. \& } \\
\text { Horadam, A. F., Editors }\end{array}$ & 37 & $11-06,11 \mathrm{~B} 37,11 \mathrm{~B} 39$ & 57 & 450 \\
\hline $\begin{array}{l}\text { Borwein, Jonathan \& } \\
\text { BorweIn, PETER }\end{array}$ & 20 & $11-00,65-00$ & 56 & 403 \\
\hline Borwein, Peter & 20 & $\begin{array}{l}\text { See: Borwein, Jonathan \& } \\
\text { Borwein, Peter }\end{array}$ & 56 & 403 \\
\hline BOWERS, K. \& LuND, J., Editors & 35 & $93-06,93 \mathrm{~B} 40,65-06$ & 57 & 448 \\
\hline Bowman, K. O. \& Shenton, L. R. & 14 & $65 \mathrm{U} 05,40 \mathrm{~A} 15,65 \mathrm{~B} 10,41 \mathrm{~A} 21$ & 56 & 398 \\
\hline Bressoud, DaVid M. & 18 & $11-01,11 \mathrm{~A} 51,11 \mathrm{Y} 05,11 \mathrm{Y} 11$ & 56 & 400 \\
\hline BULIRSCH, R. & 22 & $\begin{array}{l}\text { See: Bank, R. E., Bulirsch, R. \& } \\
\text { MERTEN, K., Editors }\end{array}$ & 56 & 405 \\
\hline $\begin{array}{l}\text { CANuto, Claudio, } \\
\text { HussainI, M. Youssuff, } \\
\text { QuARTERONI, AlFio \& } \\
\text { ZanG, ThOMAS }\end{array}$ & 43 & $65 \mathrm{M} 60,65 \mathrm{~N} 30,65 \mathrm{~N} 35$ & 57 & 876 \\
\hline $\begin{array}{l}\text { Chan, Tony F., Glowinski, Roland, } \\
\text { PeriauX, JaCQues \& } \\
\text { WidLund, OlOF B., Editors }\end{array}$ & 33 & $65-06,65 \mathrm{~N} 20,65 \mathrm{~N} 30$ & 57 & 446 \\
\hline Chua, Leon O. & 2 & $\begin{array}{l}\text { See: PARKer, Thomas S. \& ChUA, } \\
\text { LEON O. }\end{array}$ & 56 & 377 \\
\hline $\begin{array}{l}\text { ChUi, C. K., SChempP, W. \& } \\
\text { ZelleR, K., Editors }\end{array}$ & 9 & $41-06,41 \mathrm{~A} 63$ & 56 & 387 \\
\hline Cox, David A. & 19 & $\begin{array}{l}\text { 11-01, 11A41, 11D09, 11E25, 11R37, } \\
\quad 11 \mathrm{G} 15\end{array}$ & 56 & 401 \\
\hline DíAZ, J. C., Editor & 34 & $65-06$ & 57 & 447 \\
\hline $\begin{array}{l}\text { Digby, Pete, Galwey, Nick \& } \\
\text { Lane, Peter }\end{array}$ & 15 & $62-04$ & 56 & 398 \\
\hline $\begin{array}{l}\text { ELDÉN, LARS \& } \\
\text { WITTMEYER-Koch, LINDE }\end{array}$ & 39 & $65-01$ & 57 & 870 \\
\hline FLANNERY, BRIAN P. & 12 & $\begin{array}{l}\text { See: Press, William H., FlanNery, } \\
\text { Brian P., Teukolsky, Saul A. \& } \\
\text { Vetterling, William T. }\end{array}$ & 56 & 396 \\
\hline GALWEY, NICK & 15 & $\begin{array}{l}\text { See: Digby, Pete, Galwey, Nick \& } \\
\text { Lane, Peter }\end{array}$ & 56 & 398 \\
\hline
\end{tabular}




\section{Author}

GLOWINSKI, RoLAND

Golub, Gene H. \&

VAN LOAN, Charles F.

Grossman, Robert, Editor

Gunzburger, Max D.

HAYES, LINDA J.

Heiberger, Richard M.

HEINRICH, BERND

Holt, Derek F. \& Plesken, W.

Horadam, A. F.

HussaINI, M. YoussufF

KINCAID, David R. \& HAYES, LiNDA J., Editors

KOLMAN, BERNARD

KRESS, RAINER

KŔfžek, M. \& NeitTaAnMÄKi, P.

LANe, Peter

LeVeque, Randall J.

LiU, Ding-Yuan

LU, Chao

LUND, J.

McCormick, Stephen F., Editor

Merten, K.

Mittelmann, Hans D. \& Roose, DiRK, Editors

MOCKUS, JoNAS

NeitTAanMÄKI, P.

NÜRNBERGER, GÜNTHER

PARKer, ThOMAS S. \& Chua, Leon O.

Periaux, Jacques

Philippou, A. N.

Pinkus, Allan M.
Review

Number

Classification

Vol. Page

33 See: Chan, Tony F., Glowinski, Roland, Periaux, Jacques \& WidLund, Olof B., Editors $\quad 57 \quad 446$

$4 \quad 65-01,65 \mathrm{Fxx} \quad 56 \quad 380$

$36 \quad 68-02,65-02,70-04,68 \mathrm{Q} 40,65 C 99 \quad 57 \quad 449$

$\begin{array}{llll}40 & 65 \mathrm{~N} 30,76 \mathrm{D} 05 & 57 & 871\end{array}$

23 See: KincaId, David R. \& HaYes, LINDA J., Editors $56 \quad 406$

$1762-01,62 \mathrm{~J} 10,62 \mathrm{Kxx}, 65 \mathrm{Fxx} \quad 56 \quad 400$

$44 \quad 65-02,65 \mathrm{~N} 05,65 \mathrm{~N} 10,65 \mathrm{~N} 15 \quad 57 \quad 878$

32 20-02, 20D05, 20E99 $57 \quad 445$

37 See: Bergum, G. E., Philippou,

A. N. \& Horadam, A. F., Editors $57 \quad 450$

43 See: Canuto, Claudio, Hussaini, M. YousSUFF, QUARTERONI, Alfio \& ZANG, ThOMAS $\quad 57 \quad 876$

$2365-06,65 \mathrm{~F} 10 \quad 56 \quad 406$

28 See: Belinfante, Johan G. F. \& KOLMAN, BERNARD $\quad 56 \quad 886$

$345-01,45 \mathrm{~A} 05,65 \mathrm{R} 20 \quad 56 \quad 379$

$41 \quad 65-01,35 \mathrm{~A} 15,65 \mathrm{~N} 25,65 \mathrm{~N} 30 \quad 57 \quad 873$

15 See: Digby, Pete, Galwey, Nick \& LANE, PETER $\quad 56 \quad 398$

$42 \quad 65-02,35 \mathrm{~L} 65,76-08 \quad 57 \quad 875$

10 See: Su, Bu-QING \& LIU, Ding$\begin{array}{lll}\text { YUAN } & 56 & 387\end{array}$

25 See: TOLIMIERI, RICHARD, AN, MYOUNG \& Lu, ChaO

35 See: BOWERS, K. \& LUND, J., Editors

65-02, 65Mxx, 65Nxx

See: BANK, R. E., Bulirsch, R. \& MERTEN, K., Editors $65 \mathrm{H} 10,65 \mathrm{~L} 10,65 \mathrm{M} 60,58 \mathrm{C} 27$

60-02, 49D37, 60G15, 60G17, 60G35, 60G60, 62C10

41 See: KR̆fŽEK, M. \& NeitTAanMÄKI, $P$.

$2 \quad 34-01,34 \mathrm{C} 35,65 \mathrm{~L} 05,65 \mathrm{~L} 07$

56 377

33 See: Chan, Tony F., Glowinski, Roland, Periaux, Jacques \& Widlund, Olof B., Editors

37 See: Bergum, G. E., Philippou, A. N. \& HoradaM, A. F., Editors 41-02, 41A29, 41A50, 41A52, 41A65, $65 \mathrm{D} 15$ 


\begin{tabular}{|c|c|c|c|c|}
\hline Author & $\begin{array}{l}\text { Review } \\
\text { Number }\end{array}$ & Classification & Vol. & Page \\
\hline Plesken, $\mathrm{W}$. & 32 & See: Holt, Derek F. \& Plesken, W. & 57 & 445 \\
\hline Pless, Vera & 16 & $94-01,94 \mathrm{Bxx}, 05 \mathrm{~B} 05$ & 56 & 399 \\
\hline Prenter, P. M. & 7 & $65-01,65 \mathrm{D} 07,65 \mathrm{~L} 60,65 \mathrm{~N} 30$ & 56 & 384 \\
\hline $\begin{array}{l}\text { Press, William H., Flannery, } \\
\text { Brian P., TeUkOlsky, Saul A., \& } \\
\text { Vetterling, William T. }\end{array}$ & 12 & $65-04$ & 56 & 396 \\
\hline QuARteroni, Alfio & 43 & $\begin{array}{l}\text { See: Canuto, Claudio, Hussaini, } \\
\text { M. YoussufF, Quarteroni, } \\
\text { Alfio, \& Zang, Thomas }\end{array}$ & 57 & 876 \\
\hline ROOSE, DiRK & 6 & $\begin{array}{l}\text { See: Mittelmann, Hans D. \& } \\
\text { Roose, Dirk, Editors }\end{array}$ & 56 & 383 \\
\hline Rutishauser, Heinz & 38 & $65-01$ & 57 & 869 \\
\hline SCHEID, FRANCIS & 13 & $65-01$ & 56 & 397 \\
\hline SCHEMPP, W. & 9 & $\begin{array}{l}\text { See: Chui, C. K., SchempP, W. \& } \\
\text { Zeller, K., Editors }\end{array}$ & 56 & 387 \\
\hline SCHWARZ, H. R. & 1 & $65-01,65 N 30$ & 56 & 377 \\
\hline SHENTON, L. R. & 14 & $\begin{array}{l}\text { See: Bowman, K. O. \& SHENToN, } \\
\text { L. R. }\end{array}$ & 56 & 398 \\
\hline SPEDICATO, EMILIO & 5 & $\begin{array}{l}\text { See: AbafFy, Jozsef \& Spedicato, } \\
\text { Emilio }\end{array}$ & 56 & 382 \\
\hline Su, Bu-QING \& LiU, Ding-YuAN & 10 & $41-01,41 \mathrm{~A} 15,68 \mathrm{U} 05$ & 56 & 387 \\
\hline TANGORA, MARTIN C., Editor & 45 & $20-06,20-04,05-04,08-04,16-04$ & 57 & 880 \\
\hline Teukolsky, Saul A. & 12 & $\begin{array}{l}\text { See: Press, William H., Flannery, } \\
\text { Brian P., Teukolsky, Saul A. \& } \\
\text { Vetterling, William T. }\end{array}$ & 56 & 396 \\
\hline TILDESLEY, D. J. & 30 & $\begin{array}{l}\text { See: Allen, M. P. \& Tildesley, } \\
\text { D. J. }\end{array}$ & 57 & 442 \\
\hline $\begin{array}{l}\text { TOLIMIERI, RichaRd, } \\
\text { AN, Myoung \& Lu, ChaO }\end{array}$ & 25 & $94-01,65 \mathrm{~T} 05,68 \mathrm{Qxx}, 94 \mathrm{~A} 11$ & 56 & 876 \\
\hline UNITED LABORATORIES, INC. & 27 & $33-04,65 \mathrm{D} 20$ & 56 & 879 \\
\hline VAJDA, $\mathrm{S}$. & 21 & $11-01,11 \mathrm{~B} 39$ & 56 & 404 \\
\hline Van Loan, Charles F. & 4 & $\begin{array}{l}\text { See: Golub, Gene H. \& Van Loan, } \\
\text { Charles F. }\end{array}$ & 56 & 380 \\
\hline Vetterling, William T. & 12 & $\begin{array}{l}\text { See: Press, William H., Flannery, } \\
\text { Brian P., Teukolsky, Saul A. \& } \\
\text { Vetterling,William T. }\end{array}$ & 56 & 396 \\
\hline WAHBA, GRACE & 31 & $65-02,65 \mathrm{D} 07,65 \mathrm{D} 10,62-07$ & 57 & 444 \\
\hline Widlund, Olof B. & 33 & $\begin{array}{l}\text { See: Chan, Tony F., Glowinski, } \\
\text { Roland, PeriauX, JacQues \& } \\
\text { Widlund, Olof B., Editors }\end{array}$ & 57 & 446 \\
\hline WITTMEYER-KOCH, LINDE & 39 & $\begin{array}{l}\text { See: ElDEN, LARS \& WitTMEYER- } \\
\text { Koch, LINDE }\end{array}$ & 57 & 870 \\
\hline WONG, R. & 11 & $41-02,41 \mathrm{~A} 60$ & 56 & 388 \\
\hline ZANG, Thomas & 43 & $\begin{array}{l}\text { See: Canuto, Claudio, Hussaini, } \\
\text { M. YoussufF, Quarteroni, } \\
\text { Alfio \& Zang, Thomas }\end{array}$ & 57 & 876 \\
\hline ZELLER, K. & 9 & $\begin{array}{l}\text { See: ChUi, C. K., SchempP, W. \& } \\
\text { ZELLER, K., Editors }\end{array}$ & 56 & 387 \\
\hline
\end{tabular}




\section{INDEX OF REVIEWS BY SUBJECT OF WORK REVIEWED}

$\begin{array}{cccc}\text { Ruthor } & \text { Review } & & \\ \text { Number } & \text { Title } & \text { Page }\end{array}$

05-XX Combinatorics

05-04 Explicit machine computation and programs

TANGORA, Martin C., Editor

45 Computers in Algebra

$57 \quad 880$

05B05 Block designs

Pless, Vera

16 Introduction to the Theory of Error-

Correcting Codes

08-XX General mathematical systems

08-04 Explicit machine computation and programs

TANGORa, Martin C., Editor

45 Computers in Algebra

11-XX Number theory

11-00 Handbooks, dictionaries, and other reference works

BORWEIN, JONATHAN \&

20 A Dictionary of Real Numbers

Borwein, Peter

11-01 Elementary exposition; textbooks

BRessoud, David M.

Cox, David A.

VAJDA, S.

11-06 Proceedings, conferences, etc.

Bergum, G. E., Philippou, A. N. \& 37

Horadam, A. F., Editors

11 A41 Primes

Cox, David A.

19

11 A51 Factorization; primality

BRESSOUd, David M.
18 Factorization and Primality Testing

19 Primes of the Form $x^{2}+n y^{2}$ : Fermat, Class Field Theory, and Complex Multiplication

21 Fibonacci \& Lucas Numbers, and the Golden Section: Theory and Applications

Applications of Fibonacci Numbers

450

Bergum, G. E., Philippou, A. N. \& 37 Applications of Fibonacci Numbers 57 HoradaM, A. F., Editors

11B39 Fibonacci and Lucas numbers and polynomials

Bergum, G. E., Philippou, A. N. \& 37 Applications of Fibonacci Numbers 57 450 Horadam, A. F., Editors

VAJDA, $S$.

21 Fibonacci \& Lucas Numbers, and the Golden Section: Theory and Applications

$11 \mathrm{D} 09$ Quadratic and bilinear equations

Cox, David A. 19 Primes of the Form $x^{2}+n y^{2}$ : Fermat, Class Field Theory, and Complex Multiplication 


$\begin{array}{llll} & \text { Review } & \text { Title } \\ \text { Author } & \text { Number } & \text { Pol. }\end{array}$

$11 \mathrm{E} 25$ Sums of squares; other particular quadratic forms

Cox, David A.

19 Primes of the Form $x^{2}+n y^{2}$ : Fermat, Class Field Theory, and Complex Multiplication

$11 \mathrm{G} 15$ Complex multiplication and moduli of abelian varieties

Cox, DAvid A.

11 R37 Class field theory

Cox, David A.

11 Y05 Factorization

BRESSOUd, DAVID M.

18 Factorization and Primality Testing

56

11 Y11 Primality

Bressoud, David M.

Factorization and Primality Testing

56

16-XX Associative rings and algebras

16-04 Explicit machine computation and programs

TANGORa, Martin C., Editor

45 Computers in Algebra

\section{7-XX Nonassociative rings and algebras}

\section{7-01 Elementary exposition; textbooks}

Belinfante, Johan G. F. \&

Kolman, BERNARD

\section{8}

A Survey of Lie Groups and Lie Algebras with Applications and Computational Methods

17-04 Explicit machine computation and programs

Belinfante, Johan G. F. \&

Kolman, BERNARD
28 A Survey of Lie Groups and Lie Algebras with Applications and Computational Methods

\section{0-XX Group theory and generalizations}

20-02 Advanced exposition (research surveys, monographs, etc.)

Holt, Derek F. \& Plesken, W. 32 Perfect Groups

20-04 Explicit machine computation and programs

Tangora, Martin C., Editor

45 Computers in Algebra

20-06 Proceedings, conferences, etc.

TANGora, Martin C., Editor

20D05 Classification of simple and nonsolvable groups

Holt, Derek F. \& Plesken, W. 32 Perfect Groups

20E99 None of the above, but in this section

Holt, Derek F. \& Plesken, W. 32 Perfect Groups 


$$
\begin{array}{lll} 
& \text { Review } & \\
\text { Author } & \text { Number } & \text { Tolle }
\end{array}
$$

22-XX Topological groups, Lie groups

22-01 Elementary exposition; textbooks

Belinfante, Johan G. F. \& 28

A Survey of Lie Groups and Lie Algebras with Applications and Kolman, BERNARD Computational Methods

\section{3-XX Special functions}

33-04 Explicit machine computation and programs

UNITED LABORATORIES, INC.

27 Mathematical Function Library for Microsoft-FORTRAN

\section{4-XX Ordinary differential equations}

34-01 Elementary exposition; textbooks

Parker, Thomas S. \& Chua, Leon O. 2 Practical Numerical Algorithms For Chaotic Systems

34C35 Dynamical systems

Parker, Thomas S. \& Chua, Leon O. 2 Practical Numerical Algorithms For Chaotic Systems

\section{5-XX Partial differential equations}

\section{A15 Variational methods}

KŘ́ž̌E, M. \& NeitTAANMÄKI, P.

\section{L65 Conservation laws}

LeVeque, RANDALl J.

\section{0-XX Sequences, series, summability}

41 Finite Element Approximation of Variational Problems and Applications

40A15 Convergence and divergence of continued fractions

Bowman, K. O. \& Shenton, L. R. 14 Continued Fractions in Statistical Applications

\section{1-XX Approximations and expansions}

41-01 Elementary exposition; textbooks

Su, Bu-Qing \& LiU, Ding-Yuan 10 Computational Geometry: Curve and Surface Modeling 56

41-02 Advanced exposition (research surveys, monographs, etc.)

NÜRNBERGER, GÜNTHER,

Pinkus, Allan M.

WoNG, R.
24 Approximation by Spline Functions 56

8 On $L^{1}$-approximation $\quad 56$

11 Asymptotic Approximation of Integrals

41-06 Proceedings, conferences, etc.

CHUI, C. K., SCHEMPP, W. \&

9 Multivariate Approximation Theory 56 ZELLER, K., Editors

41A15 Spline approximation

Su, Bu-QING \& Liu, Ding-Yuan

10 Computational Geometry: Curve and Surface Modeling

41A21 Padé approximation

Bowman, K. O. \& Shenton, L. R. 14 Continued Fractions in Statistical Applications 
$\begin{array}{ccc}\text { Review } & \text { Tithor } & \text { Vol. Page }\end{array}$

41A29 Approximation with constraints

Pinkus, Allan M.

8 On $L^{1}$-approximation

$56 \quad 385$

41A50 Best approximation, including Chebyshev systems

Pinkus, Allan M.

8 On $L^{1}$-approximation

56

385

41A52 Uniqueness of best approximation

$\begin{array}{lllll}\text { PinkUS, Allan M. } & 8 & \text { On } L^{1} \text {-approximation } & 56 & 385\end{array}$

41A60 Asymptotic approximations, asymptotic expansions (steepest descent, etc.)

WONG, R.

11 Asymptotic Approximation of Integrals

41A63 Multidimensional problems

CHUI, C. K., SCHEMPP, W. \& $\quad 9 \quad$ Multivariate Approximation Theory $56 \quad 387$

ZELLER, K., Editors

41A65 Abstract approximation theory (approximation in normed linear spaces and other abstract spaces)

Pinkus, Allan M.

8 On $L^{1}$-approximation

56

\section{5-XX Integral equations}

45-01 Elementary exposition; textbooks

KRESS, RAINER

3 Linear Integral Equations

45A05 Linear integral equations

KRESS, RAINER

3 Linear Integral Equations

49-XX Calculus of variations and optimal control; optimization

49D37 Nonlinear programming

MockUs, JoNAS

26 Bayesian Approach to Global Optimization-Theory and Applications 56

58-XX Global analysis, analysis on manifolds

$58 \mathrm{C} 27$ Singularities of differentiable maps

Mittelmann, Hans D. \& 6 Continuation Techniques and BifurRoose, Dirk, Editors cation Problems

60-XX Probability theory and stochastic processes

60-02 Advanced exposition (research surveys, monographs, etc.)

MOCKUS, JoNAS

60G15 Gaussian processes

MockUs, JoNAS

26 Bayesian Approach to Global Optimization-Theory and Applications 56.878

60G17 Sample path properties

MOCKUS, JoNAS

26 Bayesian Approach to Global Optimization-Theory and Applications 56 
Review

Number

60G60 Random fields

Mockus, JoNAS
Title

Vol. Page

\section{2-XX Statistics}

26 Bayesian Approach to Global Optimization-Theory and Applications 56

62-01 Elementary exposition; textbooks

HEIBERGER, RichaRd M. 17 Computation for the Analysis of Designed Experiments

62-04 Explicit machine computation and programs

Digby, Pete, Galwey, Nick \& $\quad 15 \quad$ Genstat 5: A Second Course $\quad 56398$

Lane, Peter

62-07 Data analysis

WAHBA, GRACE

$31 \quad$ Spline Models for Observational Data 57

$62 \mathrm{C} 10$ Bayesian problems; characterization of Bayes procedures

Mockus, JoNAS

26 Bayesian Approach to Global Optimization-Theory and Applications 56

$62 \mathrm{~J} 10$ Analysis of variance and covariance

HEIBERGER, RichaRd M.

$62 \mathrm{Kxx}$ Experimental design

HEIBERGER, RichaRd M.

\section{5-XX Numerical analysis}

17 Computation for the Analysis of Designed Experiments

17 Computation for the Analysis of Designed Experiments

65-00 Handbooks, dictionaries, and other reference works

BorweIN, Jonathan \&

20 A Dictionary of Real Numbers

Borwein, Peter

65-01 Elementary exposition; textbooks

ELDÉN, LARS \&

WITTMEYER-KOCH, LINDE

Golub, Gene H. \&

VAN LOAN, Charles F.

KŘ́lŽé, M. \& NeitTaAnMäKi, P.

Prenter, P. M.

Rutishauser, Heinz

SCHEID, Francis

SCHWARZ, H. R.
39 Numerical Analysis: An Introduction 57

4 Matrix Computations

41 Finite Element Approximation of Variational Problems and Applications

$7 \quad$ Splines and Variational Methods 56

38 Lectures on Numerical Mathematics 57

384

869

13 Solved Problems in Numerical Analysis

1 Finite Element Methods

65-02 Advanced exposition (research surveys, monographs, etc.)

ABAFFY, JOZSEF \&

SPEDICATo, EMILIO

Grossman, Robert, Editor

HEINRICH, BERND
5 ABS Projection Algorithms: Mathematical Techniques for Linear and Nonlinear Equations

36 Symbolic Computation: Applications to Scientific Computing

44 Finite Difference Methods on Irregular Networks: A Generalized Approach to Second Order Elliptic Problems 57 


$\begin{array}{lll}\text { Author } & \text { Review } & \text { Title } \\ \text { Number } & \text { Page }\end{array}$

LEVEQUe, RANDALl J.

MCCORMICK, STEPHEN F., Editor

WAHBa, Grace
42 Numerical Methods for Conservation Laws 57

29 Multigrid Methods 57

31 Spline Models for Observational Data 57

65-04 Explicit machine computation and programs

Press, William H., Flannery, Brian P., Teukolsky, Saul A. \&

12 Numerical Recipes in Pascal-The Art VetTeRling, William T. of Scientific Computing

65-06 Proceedings, conferences, etc.

BANK, R. E., Bulirsch, R. \& Merten, K., Editors

BOWERS, K. \& LUND, J., Editors

Chan, Tony F., Glowinski, Roland, 33 PeriauX, JacQues \& Widlund, OlOF B., Editors

Díaz, J. C., Editor

KinCaID, David R. \& HAYES, LINDA J., Editors

65B10 Summation of series

Bowman, K. O. \& Shenton, L. R.

22

Mathematical Modelling and Simulation of Electrical Circuits and Semiconductor Devices

Computation and Control

Domain Decomposition Methods for Partial Differential Equations

Mathematics for Large Scale Computing

23 Iterative Methods for Large Linear Systems 406

14 Continued Fractions in Statistical Applications

65C99 None of the above, but in this section

Grossman, Robert, Editor

36 Symbolic Computation: Applications to Scientific Computing

65Dxx Numerical approximation

NÜRNBERGER, GÜNTHER,

Approximation by Spline Functions 56

65D07 Splines

Prenter, P. M.

$7 \quad$ Splines and Variational Methods 56

WAHBa, Grace

Spline Models for Observational Data 57

444

65D10 Smoothing, curve fitting

WAHBA, Grace

31 Spline Models for Observational Data

65D15 Algorithms for functional approximation

Pinkus, Allan M.

8 On $L^{1}$-approximation

65D20 Computation of special functions, construction of tables

UNITED LABORATORIES, INC.

Mathematical Function Library for Microsoft-FORTRAN

65Fxx Numerical linear algebra

Golub, Gene H. \& VAN Loan, Charles F.

HEIBERGER, RichARD M.
4 Matrix Computations 


$\begin{array}{cc}\text { Author } & \text { Review } \\ \text { Number }\end{array}$

Title

Vol. Page

$65 \mathrm{~F} 10$ Iterative methods for linear systems

AbafFy, Jozsef \& Spedicato, Emilio 5 ABS Projection Algorithms: Mathematical Techniques for Linear and Nonlinear Equations

KINCAID, David R. \&

HAYES, LiNDA J., Editors

23 Iterative Methods for Large Linear Systems

$65 \mathrm{H} 10$ Systems of equations

Abaffy, Jozsef \& Spedicato, Emilio

5 ABS Projection Algorithms: Mathematical Techniques for Linear and Nonlinear Equations

Mittelmann, Hans D. \&

RoOSE, DIRK, Editors

6 Continuation Techniques and Bifurcation Problems

$65 \mathrm{~L} 05$ Initial value problems

BANK, R. E., BuLIRSCH, R. \&

22 Mathematical Modelling and Simulation of Electrical Circuits and Semiconductor Devices

Parker, Thomas S. \& Chua, Leon O. 2 Practical Numerical Algorithms For Chaotic Systems

65L07 Numerical investigation of stability of solutions

Parker, Thomas S. \& Chua, Leon O. 2 Practical Numerical Algorithms For Chaotic Systems

$65 \mathrm{~L} 10$ Boundary value problems

Mittelmann, Hans D. \&

6 Continuation Techniques and Bifurcation Problems

65L60 Finite elements, Rayleigh-Ritz and Galerkin methods, finite methods

Prenter, P. M.

7 Splines and Variational Methods

$65 \mathrm{Mxx}$ Partial differential equations, initial value problems

McCormick, Stephen F., Editor 29 Multigrid Methods

65M60 Finite elements, Rayleigh-Ritz and Galerkin methods, finite methods

Canuto, Claudio, Hussaini,

43 Spectral Methods in Fluid Dynamics

M. YOUSSUFF, QUARTERONI,

AlFio \& ZANG, THOMAS

Mittelmann, Hans D. \&

6 Continuation Techniques and Bifur-

Roose, DiRK, Editors cation Problems

$65 \mathrm{Nxx}$ Partial differential equations, boundary value problems

MCCormick, Stephen F., Editor

29 Multigrid Methods

65 N05 Derivation of finite difference approximations

HEINRICH, BERND

44 Finite Difference Methods on Irregular Networks: A Generalized Approach to Second Order Elliptic Problems 57

$65 \mathrm{~N} 10$ Stability and convergence of difference methods

HEINRICH, BERND

65 N15 Error bounds

HEINRICH, BERND
$44 \quad$ Finite Difference Methods on Irregular Networks: A Generalized Approach to Second Order Elliptic Problems 57

44 Finite Difference Methods on Irregular Networks: A Generalized Approach to Second Order Elliptic Problems 


$$
\begin{array}{lll}
\text { Author } & \text { Review } & \text { Title } \\
\text { Number } & \text { Page }
\end{array}
$$

$65 \mathrm{~N} 20$ Solution of difference equations

Chan, Tony F., Glowinski, Roland, 33 Domain Decomposition Methods for

PeriauX, Jacques \& WidLund,

OloF B., Editors

$65 \mathrm{~N} 25$ Eigenvalue problems

KŘ́lžeK, M. \& NeitTaAnmäKi, P.

41 Finite Element Approximation of

Variational Problems and Applications

65N30 Finite elements, Rayleigh-Ritz and Galerkin methods, finite methods

Canuto, Claudio, Hussaini,

43 Spectral Methods in Fluid Dynamics

M. YOUSSUFF, QUARTERONI,

AlFio \& ZANG, THOMAS

Chan, Tony F., Glowinski, Roland, 33

Domain Decomposition Methods for

Periaux, JacQues \& Widlund, Partial Differential Equations

OlOF B., Editors

GunZburger, MaX D.

40 Finite Element Methods for Viscous Incompressible Flows: A Guide to Theory, Practice, and Algorithms 57

KŘižeK, M. \& NeitTAanmäKi, P.

Finite Element Approximation of Variational Problems and Applications

7 Splines and Variational Methods

Prenter, P. M.

1 Finite Element Methods

SCHWARZ, H. R.

65N35 Collocation and related methods

Canuto, Claudio, Hussaini,

M. YousSUfF, QUARTERONI,

43 Spectral Methods in Fluid Dynamics

AlFio \& ZANG, ThOMAS

65P05 Partial differential equations, miscellaneous problems

BANK, R. E., BULIRSCH, R., \& MERTEN, K., Editors

$65 \mathrm{R} 20$ Integral equations

KRESS, RAINER

22 Mathematical Modelling and Simulation of Electrical Circuits and Semiconductor Devices

3 Linear Integral Equations

65T05 Harmonic analysis and synthesis

Tolimieri, RichaRd, AN, Myoung \& 25 Algorithms for Discrete Fourier

Lu, ChaO Transform and Convolution

65U05 Numerical methods in probability and statistics

Bowman, K. O. \& Shenton, L. R. 14 Continued Fractions in Statistical

Applications 56

\section{8-XX Computer science}

68-02 Advanced exposition (research surveys, monographs, etc.)

Grossman, ROBERT, Editor

36 Symbolic Computation: Applications

to Scientific Computing

68Qxx Theory of computing

Tolimieri, RichaRd, AN, Myoung \& 25 Algorithms for Discrete Fourier Lu, ChaO Transform and Convolution 


$\begin{array}{lll} & \text { Review } & \\ \text { Author } & \text { Number } & \text { Vol. }\end{array}$

68Q40 Symbolic computation, algebraic computation

Grossman, Robert, Editor $\quad 36$ Symbolic Computation: Applications to Scientific Computing $\quad 57 \quad 449$

68U05 Computer graphics; computational geometry

Su, Bu-Qing \& LiU, Ding-YuAN 10 Computational Geometry: Curve and Surface Modeling

70-XX Mechanics of particles and systems

70-01 Elementary exposition; textbooks

Allen, M. P. \& Tildesley, D. J. $\quad 30 \quad$ Computer Simulation of Liquids $\quad 57 \quad 442$

70-04 Explicit machine computation and programs

Allen, M. P. \& Tildesley, D. J. $\quad 30 \quad$ Computer Simulation of Liquids $\quad 57 \quad 442$

Grossman, Robert, Editor $\quad 36$ Symbolic Computation: Applications

to Scientific Computing $\quad 57 \quad 449$

76-XX Fluid mechanics

76-08 Computational methods

LeVeque, Randall J. 42 Numerical Methods for Conservation

Laws 57

76D05 Navier_Stokes equations

GunzBurger, Max D. $\quad 40$ Finite Element Methods for Viscous

Incompressible Flows: A Guide to

Theory, Practice, and Algorithms 57

82-XX Statistical physics, structure of matter

82-01 Elementary exposition; textbooks

Allen, M. P. \& Tildesley, D. J. $\quad 30 \quad$ Computer Simulation of Liquids $\quad 57 \quad 442$

82-04 Explicit machine computation and programs

Allen, M. P. \& Tildesley, D. J. $\quad 30 \quad$ Computer Simulation of Liquids $\quad 57 \quad 442$

82A50 Liquids

Allen, M. P. \& Tildesley, D. J. $\quad 30 \quad$ Computer Simulation of Liquids $\quad 57 \quad 442$

93-XX Systems theory; control

93-06 Proceedings, conferences, etc.

Bowers, K. \& Lund, J., Editors $\quad 35 \quad$ Computation and Control $\quad 57 \quad 448$

93B40 Computational methods

Bowers, K. \& Lund, J., Editors $\quad 35 \quad$ Computation and Control $\quad 57 \quad 448$

94-XX Information and communication, circuits

94-01 Elementary exposition; textbooks

Pless, Vera 16 Introduction to the Theory of Error-

Correcting Codes 56

Tolimieri, Richard, AN, Myoung \& 25 Algorithms for Discrete Fourier

$\begin{array}{llll}\mathrm{Lu}, \mathrm{CHAO} & \text { Transform and Convolution } & 56 & 876\end{array}$

94A11 Application of orthogonal functions in communication

Tolimieri, Richard, AN, Myoung \& 25 Algorithms for Discrete Fourier

Lu, CHAO $\quad$ Transform and Convolution $\quad 56 \quad 876$ 
94Bxx Algebraic theory of error-correcting codes

Pless, Vera

16 Introduction to the Theory of ErrorCorrecting Codes

\section{INDEX OF ERRATA}

No.

Author

Title

Vol. Page

613 Kumar, RavindRa \& JAIN, M. K. Quadrature Formulas for SemiInfinite Integrals

\section{INDEX OF SUPPLEMENTS}

\author{
DIAZ Y DIAZ, F.$$
\text { Author }
$$

Nochetto, R. H., PaOlini, M. \& VERDI, C.

LAsiecka, I. \& TRIgGiani, R.
Title

Supplement to "A table of totally real quintic number fields"

Supplement to "An adaptive finite element method for two-phase Stefan problems in two space dimensions. Part I: Stability and error estimates"

Supplement to "Numerical approximation of algebraic Riccati equations for abstract systems modelled by analytic semigroups, and applications"
Vol. Issue

56 April

57 July

57 Oct.

\section{INDEX OF MICROFICHE SUPPLEMENTS}

Author

Doll, Helmut \& Hoste, Jim

DOUGHeRTY, RANDALL \&

JaNwa, HeEralal
Title

A tabulation of oriented links

Covering radius computations for binary cyclic codes
Vol. Issue

57 Oct.

57 July 